山्山FFRANÇAISE

$\supset \mathrm{DE}$

$\stackrel{w 1}{\simeq}$ PÉDAGOGIE

\section{Revue française de pédagogie}

Recherches en éducation

$185 \mid 2013$

L'enseignement de la musique, entre institution scolaire et conservatoires

\title{
La sensibilité musicale saisie par la forme scolaire. L'éducation musicale au collège, de formalisme en formalisme
}

Sensitivity to music taken into account by school curricula. Music education in middle school from formalism to formalism

\section{Florence Eloy}

\section{(2) OpenEdition}

Journals

Édition électronique

URL : http://journals.openedition.org/rfp/4327

DOI : $10.4000 /$ rfp. 4327

ISSN : 2105-2913

Éditeur

ENS Éditions

Édition imprimée

Date de publication : 31 décembre 2013

Pagination : 21-34

ISBN : 978-2-84788-523-1

ISSN : 0556-7807

\section{Référence électronique}

Florence Eloy, « La sensibilité musicale saisie par la forme scolaire. L'éducation musicale au collège, de formalisme en formalisme », Revue française de pédagogie [En ligne], 185 | 2013, mis en ligne le 31 décembre 2016, consulté le 19 avril 2019. URL : http://journals.openedition.org/rfp/4327 ; DOI : $10.4000 /$ rfp.4327 


\section{La sensibilité musicale saisie par la forme scolaire. L'éducation musicale au collège, de formalisme en formalisme}

Florence Eloy

Cet article vise à analyser le développement, à partir des années 1970, de la référence à la sensibilité des élèves - considérée comme point de départ incontournable de toute démarche d'apprentissage musical - dans les textes officiels de l'éducation musicale au collège. Moins qu'une remise en cause de la "forme scolaire", l'appel à la sensibilité signale bien davantage une variation de cette dernière, puisque le rapport "scriptural-scolaire" au monde qu'elle suppose est mis au centre des apprentissages, l'objectif étant de transmettre des modes d'écoute "cultivés " de la musique très proches de la "disposition esthétique » décrite par Pierre Bourdieu dans La Distinction.

Mots-clés (TESE) : jeunesse, musique, éducation artistique, réforme des programmes d'études, objectifs pédagogiques, processus d'apprentissage.

Cet article s'appuie sur un constat : le développement notable, depuis la fin des années 1970, de la référence à la sensibilité des élèves dans les textes officiels de l'éducation musicale au collège. Plus précisément, cette référence est présentée comme le point de départ incontournable de toute démarche d'apprentissage musical, ce dont est emblématique la formule énoncée dans les programmes de 1977 comme de 1985, "sentir d'abord, comprendre ensuite, apprendre enfin " (Ministère de l'Éducation nationale, 1977a, 1985). Cette conception des cheminements cognitifs à adopter, loin de ne concerner que l'éducation musicale au collège, s'inscrit plus largement dans le cadre d'une prise en compte accrue, dans les curricula, de l'expérience ordinaire des élèves, étroitement liée à une volonté de rendre les processus d'apprentissage plus actifs et les savoirs plus concrets dans le contexte du collège massifié et de la diffusion de pédagogies inspirées par les principes de l'«éduca- tion nouvelle "1 (Bautier \& Rochex, 1997; Bonnéry, 2007; Harlé, 2003; Lahire, 1993; Rochex \& Crinon, 2011).

II s'agira donc, à partir de l'exemple du curriculum de l'éducation musicale et du rôle important qu'il assigne à la sensibilité des élèves, de s'interroger sur la portée de ces évolutions : qu'indiquent-elles quant au renouvellement des processus de transmission scolaire? Opèrentelles une rupture par rapport aux caractéristiques associées à la «forme scolaire» (Vincent, Lahire \& Thin, 1994; Lahire, 1993)? En effet, la forme scolaire repose sur - et cherche à transmettre - un rapport distancié au langage et au monde (un rapport "scriptural-scolaire » au langage et au monde, pour reprendre les termes de B. Lahire) ${ }^{2}$, qui semble entrer en tension avec le recours à l'expérience ordinaire des élèves, en bonne partie ancrée dans la pratique. 
L'intérêt de se focaliser, pour répondre à ces questions, sur une discipline peu centrale dans les curricula ${ }^{3}$, est multiple. Tout d'abord, la faible légitimité scolaire de la matière - faisant d'elle le support de savoirs souvent considérés comme «gratuits »-, combinée à la place centrale de la musique dans les pratiques culturelles juvéniles ${ }^{4}$, limite de manière très significative le pouvoir d'imposition symbolique de l'école dans le domaine musical, les schèmes de perception et d'appréciation des élèves en matière de musique étant en grande partie forgés en dehors du champ scolaire, par des instances de légitimation potentiellement concurrentes de l'école, telles que la famille, les médias, les industries culturelles ou encore les groupes de pairs (Lahire, 2004; Pasquier, 2005). Constituant à cet égard un «cas limite", l'enseignement de la musique au collège est tout particulièrement confronté à la question de la prise en compte de l'expérience ordinaire des élèves.

Traiter ces questions à travers l'exemple de l'enseignement de la musique au collège invite en outre à dépasser les frontières entre grands domaines de la sociologie, afin d'être en mesure de saisir des logiques relevant simultanément de différents champs ou sous-champs sociaux (Bourdieu, 2004; Lahire, 2005). En effet, pour comprendre les évolutions du curriculum de la discipline, il est indispensable de "sortir de l'école" et de se pencher sur les évolutions culturelles durant la période considérée (19382008), relevant aussi bien du domaine de la sociologie de la jeunesse que de la sociologie de la culture : d'une part, le développement très important des pratiques d'écoute musicales juvéniles dans les années 1960, qui vient modifier le poids respectif des différents prescripteurs en matière de musique, au détriment de l'école (Bantigny, 2008); d'autre part, les «métamorphoses de la distinction" (Coulangeon, 2011), autrement dit l'évolution des modes de consommation culturelle socialement considérés comme "cultivés" et dignes d'être transmis (Bourdieu \& Passeron, 1964; Bourdieu, 1967, 1979).

Cette réflexion s'appuiera essentiellement sur un corpus composé des textes officiels de l'enseignement musical au collège depuis 1938, qu'il s'agisse des programmes au sens strict du terme ou des textes d'accompagnement des programmes donnant des exemples de séquences de cours qui illustrent les cheminements intellectuels attendus des élèves ${ }^{5}$. Le travail réalisé sur ce matériau consiste en une analyse qualitative centrée sur les contenus d'enseignement et les indications relatives aux manières de les appréhender avec les élèves. II s'agira tout d'abord de décrire les manifestations de cette ouverture du curriculum à la sensibilité et à la subjectivité des élèves, conco- mitante d'une prise de distance avec la «théorie musicale». Nous montrerons ensuite qu'à travers cette évolution s'affirme un autre type de regard formel sur la musique, basé sur l'intériorisation de dispositions cultivées à l'égard de l'écoute musicale.

\section{«SENTIR D'ABORD, COMPRENDRE ENSUITE, APPRENDRE ENFIN »}

À partir de la fin des années 1970 , les références à l'expérience ordinaire des élèves en matière de musique se multiplient dans les textes officiels de l'enseignement de la musique au collège, et ce de plusieurs manières : non seulement par une ouverture du curriculum aux préférences musicales des élèves ${ }^{6}$, mais également par l'incitation à la prise en compte de leur sensibilité, de leurs manières d'écouter et de penser la musique. Ainsi, la prégnance du code musical est progressivement remise en question dans le cadre d'une injonction croissante à mobiliser la sensibilité des élèves.

\section{L'élève et sa culture placés au centre du curriculum}

Avant d'aborder plus précisément la multiplication des références à la sensibilité des élèves dans les textes officiels au cours de la période étudiée, il convient de préciser deux évolutions plus globales qui ont modifié le contexte dans lequel intervient l'enseignement musical. Les évolutions pédagogiques rattachées au «puérocentrisme" (Rayou, 2000), ainsi que le développement des pratiques d'écoute de musique enregistrée chez les élèves, ont en effet transformé profondément le positionnement des programmes officiels vis-à-vis des élèves, programmes qui, à partir des années 1970, dans un contexte de diversification progressive du recrutement social de l'enseignement secondaire, enjoignent de plus en plus les enseignants à s'appuyer sur les pratiques culturelles juvéniles dans les processus d'apprentissage. $C$ 'est ce que suggèrent $A$. Barrère et $F$. Jacquet-Francillon (2008) : “Plus l'“élève global”, c'est-à-dire l'enfant et l'adolescent, est reconnu comme tel à l'intérieur de l'école, ce que rappellent les textes officiels depuis une vingtaine d'années, plus il y amène un ensemble de désirs et d'usages (musiques, télévision, ordinateur, vêture, etc.) qui avaient toujours été étrangers voire exclus des institutions éducatives ".

L'ouverture à la culture des élèves est ainsi encouragée par la diffusion d'un modèle éducatif puérocentré, qui, se 


\section{Présentation du corpus}

Les instructions officielles de l'éducation musicale retenues pour ce corpus remontent jusqu'à la fin des années 1930 (1938). Cette borne temporelle a été retenue car c'est avec les textes de 1938 que l'enseignement scolaire de la musique se généralise à l'ensemble du premier cycle du secondaire, y compris les écoles primaires supérieures et les cours complémentaires. En effet, systématique dans les établissements féminins, cette discipline n'est, avant 1938, obligatoire pour les garçons durant tout le cycle qu'à de courtes périodes. Ces textes, qui émanent du ministère de l'Éducation nationale, sont au nombre de vingt-deux répartis en sept "générations de programmes" (les textes sont publiés de manière fragmentée, les différents niveaux du premier cycle du secondaire faisant par exemple l'objet de publications différentes et n'intervenant pas au même moment), et ont des statuts différents. Ce corpus réunit ainsi des arrêtés ministériels et des circulaires (ces dernières sont des compléments de programmes qui concernent soit uniquement l'éducation musicale soit l'enseignement des arts en général).

L'analyse de ce corpus est de nature qualitative. Ainsi, nous n'avons pas procédé à une analyse de contenu de nature quantitative, comme a pu le faire V. Isambert-Jamati (1970) sur les discours de distribution de prix au lycée. Un des éléments majeurs plaidant selon nous pour l'adoption de cette modalité de traitement des données qualitatives est relatif à l'instabilité du corpus selon les périodes. II est donc difficile de comparer les termes employés par les textes "toutes choses égales par ailleurs".

Pour résumer ces évolutions, on peut tout d'abord évoquer la variation importante des activités recouvertes par la discipline : en schématisant (les vocables assignés à chacune des activités évoluant au cours du temps, ce qui complique l'effort de synthèse), le programme de 1938 fait la part belle au chant, qui permet d'aborder à la fois la technique vocale et des notions de solfège (abordées aussi à travers les dictées et les lectures de notes). L'histoire de la musique constitue l'autre pan du programme, avec une partie théorique et une partie destinée à l'écoute des œuvres. Cette configuration reste sensiblement la même jusqu'aux textes de 1977-1978, même si s'opère un certain rééquilibrage au profit de l'histoire de la musique et de l'écoute, qui font l'objet de prescriptions plus détaillées dans les instructions des années 1940 et 1960 que dans le programme de 1938. Le programme de 1977 introduit des changements importants du point de vue de l'organisation de la discipline, avec la disparition des rubriques "solfège» et «histoire de la musique» et l'apparition de la pratique instrumentale. Les instructions de 1985, quant à elles, prescrivent de manière inédite les activités de création et d'invention, même si un paragraphe est déjà consacré à la «création personnelle» dans la circulaire de 1977 portant sur les disciplines artistiques. Ce n'est qu'avec le texte de 2008 que cette configuration se modifie, la pratique instrumentale n'apparaissant plus comme une activité à part entière de l'éducation musicale (bien qu'elle puisse être abordée ponctuellement à travers les «projets musicaux»).

Enfin, autre évolution remarquable, la présentation des directives - qui se résument jusqu'aux années 1960 en bonne partie à une énumération des contenus d'enseignement à transmettre aux élèves (techniques de chant, notions de solfège, périodes ou compositeurs) - devient tout autre par la suite : les rédacteurs des programmes insistent alors bien davantage sur la conception à défendre quant aux objectifs de l'éducation musicale, et sur les méthodes à appliquer pour la mettre en œuvre dans chaque activité. Par conséquent, on assiste à une tendance «inflationniste» de la longueur des textes (celle-ci allant d'une page pour le programme de 1938 à une vingtaine de pages pour le programme de 2008), les instructions officielles prenant alors la forme de longs textes rédigés - au besoin agrémentés de schémas, comme c'est le cas pour le programme de 2008 - détaillant la manière dont les œuvres doivent être présentées aux élèves ainsi que les compétences d'écoute et de pratiques de la musique à transmettre.

nourrissant des théoriciens et des expériences de l'«éducation nouvelle" et trouvant également des prolongements à travers les théories développées par les défenseurs de la "cause des enfants", s'impose progressivement dans les années 1970 (Rayou, 2000). Dépassant les antagonismes entre des acteurs éducatifs très divers, cette conception pédagogique est progressivement adoptée par le ministère de l'Éducation nationale. La loi d'orientation sur l'éducation de 1989, impulsée par Lionel Jospin alors ministre de l'Éducation nationale, entérine définitivement l'adoption de cette conception de l'éducation par le système éducatif français, à travers le mot d'ordre de «l'élève au centre". Selon ce texte, c'est dorénavant l'école qui doit s'adapter à l'élève, et non plus l'inverse.
Cette conception puérocentrée transparaît très clairement dans les instructions officielles de l'éducation musicale à partir des années 1970. Cela se manifeste, entre autres, par la multiplication des références à l'élève et à l'enfant, mise en évidence par O. Tripier-Mondancin (2008, p. 204-206). L'attention croissante portée à l'élève se décline plus particulièrement à travers la contribution souhaitée de l'éducation musicale au développement de la personnalité de l'enfant, qui reprend la perspective défendue par les tenants de l'éducation nouvelle. C'est ainsi que R. Cousinet conçoit l'acte éducatif comme une "attitude d'acceptation de l'enfance en tant que telle, reconnaissance de la valeur de l'enfance comme une période nécessaire dans le développement de l'homme " 
(Cousinet, cité par Rayou, 2000, p. 248). L'apparition de la pratique instrumentale (avec les textes de 1977-1978) puis celle des activités d'« invention et de création " (avec le programme de 1985) entrent tout à fait dans cette logique, le cours de musique devant constituer un espace d'expression pour l'élève, dans le cadre duquel est stimulée sa créativité. Le développement de la personnalité de l'enfant à travers l'expression artistique devient ainsi un objectif prioritaire de l'enseignement scolaire de la musique à partir des textes de 1977-1978 :

\begin{abstract}
L'éducation artistique joue un rôle considérable dans la formation globale de l'individu, en mettant en jeu des capacités créatives qui risqueraient sans cela de rester en partie inemployées et en intervenant comme élément de développement de la personnalité [...]. L'éducation artistique doit être tout à la fois une formation par l'art et une formation à l'art : formation par l'art, dans la mesure où l'objectif à atteindre concerne les composantes mêmes de la personnalité de l'élève et notamment sa sensibilité, sa créativité. [...] On peut imaginer que cette éducation esthétique se caractérise par l'aspect global de son action sur la personnalité de l'élève, se révélant ainsi sensiblement différente de ce que furent autrefois les enseignements traditionnels de la musique et du dessin (Ministère de l'Éducation nationale, 1977b)7.
\end{abstract}

Se démarquant explicitement des programmes précédents, les textes de 1977-1978 mettent au premier plan l'apport que constituent les enseignements artistiques pour le développement de la personnalité de l'élève. Ce faisant, ils reflètent l'orientation que voulait donner aux programmes l'inspecteur général de l'époque, Marcel Landowski, fervent partisan des méthodes associées à l'«éducation nouvelle »8. Cette évolution s'inscrit par ailleurs dans des tendances curriculaires plus larges, rejoignant celles soulignées par la littérature sociologique à d'autres niveaux du système éducatif, par exemple le tournant «expressif-créatif » mis en exergue par É. Plaisance dans l'enseignement maternel (Plaisance, 1986).

Cet objectif implique une prise en compte a minima des rapports à la musique et des goûts développés dans la sphère des pratiques culturelles ordinaires. C'est d'autant plus le cas que l'on assiste, à partir des années 1960, au «boom musical» porté par les jeunes générations (Donnat, 1994), qui s'intensifie dans les décennies suivantes avec l'apparition de nouveaux supports d'écoute de musique enregistrée (cassettes, CD, puis supports numériques via les lecteurs MP3) et le développement des industries culturelles, dont des segments entiers sont explicitement dédiés à la jeunesse (Sohn, 2001). Les rapports à la musique du public auquel s'adresse l'enseignement musical évoluent donc considérablement entre l'immédiat après-guerre et les années 1960 et 1970 . Les élèves deviennent progressivement des auditeurs assidus, dont les préférences musicales sont susceptibles d'entrer en contradiction avec celles dictées par le curriculum de l'éducation musicale. II devient dès lors très difficile pour les rédacteurs des programmes d'ignorer ces pratiques et ces goûts juvéniles en matière de musique, dont les programmes de 1985 prennent explicitement acte :

Le développement des techniques d'enregistrement et l'industrie du disque et de la cassette marquent forcément la pratique de l'écoute des œuvres au collège. La plupart des élèves possèdent ou peuvent posséder des disques ou des cassettes (Ministère de l'Éducation nationale, 1985) ${ }^{9}$.

Par conséquent, les allusions au "répertoire actuel» et aux «goûts des élèves » se multiplient dans les textes officiels. C'est ce que l'on peut constater à travers l'exemple du programme de 1985, qui incite les enseignants à s'appuyer sur des œuvres en prise avec l'actualité musicale, en insistant sur les modes de diffusion qui les caractérisent :

Il est donc normal que le professeur prenne en considération le répertoire actuel. Le champ est suffisamment vaste pour qu'il y trouve un accord entre les goûts de son temps et ses propres objectifs. Ces références fondamentales comprennent la diffusion ordinaire des disques et des cassettes, les programmes musicaux radiodiffusés et les programmes des concerts et festivals. [...] II importe que l'initiation à la culture musicale donnée par le collège garde une communication directe avec la musique entendue au-dehors [et réponde] à la diversité des attentes et des besoins individuels des élèves (Ministère de l'Éducation nationale, 1985).

La prise en compte des univers musicaux des élèves et l'ouverture du répertoire de la discipline se prolongent dans les textes des années 1990 et 2000 , dans lesquels les incitations à être attentif aux préférences musicales des élèves sont elles aussi présentes, notamment à l'occasion des activités de chant et de création ${ }^{10}$ :

L'interprétation d'un répertoire diversifié, intégrant le goût des adolescents pour la chanson contemporaine constitue la base des activités vocales [...]. La création de chanson permet aux élèves de s'exprimer avec leurs mots, avec leurs musiques tout en intégrant les acquis du cours d'éducation musicale (Ministère de l'Éducation nationale, 1997a).

Le contexte dans lequel se développent les références à la sensibilité des élèves dans le curriculum de l'éducation musicale au collège est donc marqué par un décloisonnement de la culture scolaire, comme cela a 
pu être souligné concernant d'autres pans du système éducatif (Bautier \& Rayou, 2009; Bautier \& Rochex, 1997; Bonnéry, 2007; Rochex \& Crinon, 2011). Ce faisant, l'élève est pris en compte aussi bien sous un angle strictement scolaire que de manière plus globale, l'attention étant portée également sur sa «personnalité » et sur ses pratiques culturelles extra-scolaires.

\section{Le code et le sensible : à la recherche d'une approche pratique de la musique}

Dans le cadre de ces objectifs et de cette conception de l'élève, l'approche des activités musicales préconisée par les textes officiels, qu'il s'agisse de l'écoute ou de la pratique vocale et instrumentale, évolue considérablement. Durant la première moitié du $x x^{e}$ siècle, l'enseignement de la musique dans le secondaire accorde une grande importance à la maîtrise de la codification de la musique. La prégnance de la théorie musicale se manifeste clairement dans les premiers programmes de notre corpus - ceux de 1937-1938 et de 1944 en particulier qui prescrivent l'apprentissage de quantité de notions de solfège (à travers des exercices de lecture, de dictée et de chant). Voici par exemple le programme de solfège prévu pour le niveau $6^{\circ}$ en 1944 :

Audition, chant lecture; Solfège à une et à deux voix

10 L'accord parfait de do, de fa, de sol; Exercices polyphoniques; Audition (extraits oraux); Lecture des notes et des accords parfaits, durées et silences correspondants; Mesures 2/4, 3/4, 4/4

2० La gamme de do

Tons et demi-tons, les tierces, audition (exercices oraux), chant et lecture; Nouvelles figures de durées; La liaison

3० Bémol, la gamme de fa (Ministère de l'Éducation nationale, 1944).

Au cours de la deuxième moitié du xxe siècle, le "sensible », la «sensation » ou encore l'« imagination » sont de plus en plus sollicités pour appréhender la musique. C'est à partir de la série de programmes de la fin des années 1970 - qui explicitent, bien davantage que les textes précédents, la manière dont les enseignants doivent aborder les œuvres avec les élèves - que s'exprime pleinement ce souci de ne pas mettre au premier plan l'étude théorique de la structure musicale, à travers la référence à la "sensibilité " de l'élève. Cette injonction se traduit bien dans les directives concernant les activités d'écoute de la génération de programmes de 19951998, qui est probablement celle qui va le plus loin dans cette logique de la «primauté du sensible»11:

Une [première] approche globale et sensible de la musique est privilégiée : répartition spatiale et temporelle des évènements sonores (masses, lignes, horizontalité, verticalité, logique des rapports), couleurs (familles d'instruments d'abord, timbres individuels ensuite, combinaisons), dynamiques (accents, nuances, contrastes, silences) (Ministère de l'Éducation nationale, 1995).

Une première écoute [de l'œuvre étudiée] privilégiera la sensibilité et l'imagination en s'attachant à la globalisation des éléments sonores. [...] Elle amène l'élève à s'imprégner du climat de l'œuvre sans souci prématuré d'analyse ou d'identification. À l'issue de cette première écoute, les élèves seront invités à s'exprimer de manière sensible sur des modes variés (verbe, geste, mouvement, image, dessin, graphisme, induits éventuellement par une consigne initiale). [...] Réagir, nommer des émotions : ce premier niveau du commentaire d'œuvres musicales est subjectif (Ministère de l'Éducation nationale, 1997b).

Ces extraits insistent tous deux sur l'importance d'aborder les morceaux étudiés lors des activités d'écoute sans recours, dans un premier temps, à des notions de théories musicales : l'élève doit en premier lieu retirer une impression globale du morceau. $\mathrm{Si}$, dans le premier extrait, il s'agit tout de même d'en identifier les principales caractéristiques formelles à travers des notions voulues intuitives, comme «la répartition spatiale et temporelle des évènements sonores", ou encore «la couleur » du morceau, le second extrait va plus loin dans cette perspective, invitant les enseignants à s'en tenir au registre de l'émotion et du subjectif dans cette première phase de l'écoute.

L'impératif du primat du sensible est d'autant plus explicité pour la pratique vocale et instrumentale que ces activités ont davantage partie liée avec l'usage de la théorie musicale, et notamment de la partition. Les concernant, les rédacteurs des programmes sont donc contraints d'expliciter les manières de concilier une approche sensible avec l'utilisation d'éléments de langage musical, et notamment ceux relatifs au solfège. On retrouve à leur propos la même démarche encourageant une première phase d'«imprégnation », durant laquelle l'usage du code est présenté comme contre-productif et comme un procédé pédagogique pouvant contribuer à inhiber les capacités expressives des élèves :

Lorsque les élèves ont mémorisé le premier couplet et le refrain, la partition peut être distribuée puis commentée. En effet, la distribution prématurée d'un document visuel crée une diversion très dommageable à la qualité expressive et à la rapidité de l'apprentissage : l'écoute s'en trouve manifestement émoussée et la cohésion du groupe classe a tendance à se désagréger, chacun se réfugiant derrière sa partition. [...] Elle [la partition] 
s'intègre à un cheminement pédagogique commun à l'ensemble des activités du cours d'éducation musicale, cheminement caractérisé par la primauté et la prégnance de l'écoute et de l'oral (Ministère de l'Éducation nationale, 1998).

II s'agit donc d'éviter dans un premier temps le «jargon » du solfège, dans la continuité du point de vue sur la théorie musicale développé dans les programmes dès la fin des années 1970. Dans cette perspective, la question du langage musical ne doit pas être abordée indépendamment des œuvres étudiées. Non seulement il faut privilégier dans un premier temps le "sensible» et ne pas partir de la théorie musicale ou de considérations techniques, mais, de surcroît, le solfège ne doit plus constituer un objectif d'enseignement en soi. Les notions techniques ne sont pas pour autant bannies des programmes, mais elles sont de plus en plus considérées comme des moyens, ou mieux comme des outils de l'éducation musicale, et non comme une fin en soi. Cette conception, si elle imprègne les instructions officielles à partir de la fin des années 1970, est exprimée de manière particulièrement claire dans le programme de 1985 :

Les activités de codage et de décodage ne doivent être abordées, pour chacune des notions [du langage musical] considérées, qu'au terme d'une phase d'imprégnation, de pratique et d'analyse absolument indispensable et prioritaire [...]. La technicité des codes n'est pas une fin en soi. C'est une convention commode qui permet au compositeur et à l'interprète de s'investir [...]. La musique est par essence un art abstrait. Mais c'est aussi un art immédiat. La difficulté de la pédagogie musicale tient à ce paradoxe apparent. L'éducation musicale doit donc combiner ces approches qui appartiennent à deux ordres différents : un ordre de codes et d'analyse et un ordre de perception immédiate et intuitive. L'activité musicale réelle constitue le point de départ obligé pour toute acquisition d'ordre sensoriel, sensible ou cognitif (Ministère de l'Éducation nationale, 1985).

À travers ce positionnement des textes officiels vis-àvis du solfège, tout se passe comme s'il s'agissait de mettre en suspens la forme scolaire et le rapport scriptural-scolaire dans la phase d'«approche» des œuvres étudiées, pour privilégier un apprentissage par imitation, ou encore "par corps", pour reprendre le terme de P. Bourdieu (1980), qui se passerait, à ce premier stade d'imprégnation, de savoirs objectivés par l'écrit. Les textes de 1995-1998 explicitent particulièrement ce type de démarches pédagogiques qui ont l'ambition de se fonder sur la pratique et l'incorporation, comme l'illustre cet extrait à propos de la pratique instrumentale :
Les méthodes d'apprentissage sont fondées sur la transmission orale et la perception globale et immédiate du langage. Elles favorisent la spontanéité et l'aisance tout en constituant un moyen de parvenir à l'improvisation. Ainsi, le geste instrumental réalise-t-il un son intérieur dont l'existence est liée au chant ou à une expression verbale (par exemple, les onomatopées pour les apprentissages rythmiques) qui le précède et oblige-t-il l'élève à penser les sons, les phrases, avant de les réaliser à l'instrument (Ministère de l'Éducation nationale, 1977a).

La tension existant entre, d'une part, la volonté d'adopter des modes oraux, pratiques et intuitifs de transmission, et, d'autre part, l'ambition de transmettre une approche formelle de la pratique musicale - signalée par l'injonction à "penser les sons", ou encore l'évocation des "phrases " - est manifeste dans cet extrait. En effet, l'idée de "penser les sons " peut être assimilée à un procédé de distanciation et à une attitude réflexive, tandis que la notion de "phrase » implique un découpage de la musique, deux manières d'envisager la pratique caractéristiques de la logique scripturale-scolaire. Les exemples de séquences de cours proposés aux enseignants dans les documents d'accompagnement des programmes précisent cette aspiration à concilier le sensible et le code dans la pratique musicale, à l'instar de cette activité de cours intitulé "pratique rythmique " autour d'un extrait de La Passion selon Saint-Jean de JeanSébastien Bach :

Concevoir une pédagogie du rythme à partir de diverses percussions, c'est viser un double but : rendre au corps de l'enfant et de l'adolescent sa liberté première, qui est la possibilité de dissocier les mouvements des différentes parties du corps [...]. Apprendre à analyser, puis à noter les différentes cellules composant ces motifs dans une deuxième phase de cette activité, une fois ces diverses séquences totalement intégrées par le corps, sans aucune intervention de notions théoriques, pendant ce temps d'assimilation corporelle (Ministère de l'Éducation nationale, 1998).

Dans cet extrait également, les textes décrivent une démarche dont les visées ne sont pas dénuées d'ambiguïté. Ils visent ainsi la «liberté première» de l'adolescent, donc une forme de spontanéité postulée chez l'élève, à travers une dissociation des mouvements des différentes parties du corps au contraire très liée à un rapport scriptural-scolaire au savoir, fondé sur la décomposition des pratiques ou des savoirs faisant l'objet de la transmission. Cet exemple souligne particulièrement la dualité de l'approche musicale proposée dans les textes officiels, qui doit être à la fois intuitive et intellectualisée, pratique et réflexive, tout en affirmant la primauté de cette première dimension sur la seconde. À travers la priorité accordée 
à la pratique et au sensible - renvoyant à l'expérience ordinaire des élèves - est recherchée une «mise en activité » de ces derniers afin de «donner du sens » aux apprentissages et de les rendre plus attractifs ${ }^{12}$. Ces conceptions pédagogiques tranchent avec les opérations de «mémorisation-restitution » (Johsua, 1999) sous-tendant les textes les plus anciens de notre corpus, que commente ainsi un acteur central de la rédaction des programmes, interrogé dans le cadre de cette recherche : «On voit des définitions, on voit des photocopies sur les compositeurs, sur les œuvres, bon. Et tout ça, ça ne garantit absolument rien sur le sens que ça peut avoir pour l'élève. »

La place centrale donnée à la sensibilité des élèves s'inscrit donc dans un processus d'ouverture de l'école à des formes culturelles qui lui sont extérieures. À cet égard, on peut reprendre la distinction opérée par B. Bernstein (1975) entre «code série » et «code intégré » pour décrire cette évolution : de la compartimentation rigide entre savoirs (entre les différentes disciplines, mais aussi entre les savoirs relevant de la sphère scolaire et les expériences non scolaires) caractérisant le «code série», le curriculum a évolué vers des formes et des modalités beaucoup plus souples de classifications, notamment celle distinguant culture scolaire et «culture libre», dont l'agencement relève ainsi davantage du «code intégré» à partir des années 1970. Dans cette mesure, on peut souligner la plus grande porosité de la forme scolaire par rapport à des répertoires culturels autres que ceux relevant des références canoniques, auparavant au centre du curriculum ${ }^{13}$.

\section{LA TRANSMISSION D'UN «SAVOIR-ÉCOUTER» : UNE MODALITÉ D'APPROCHE FORMELLE DE LA MUSIQUE}

Néanmoins, cette tension entre approche sensible et transmission d'un regard formel sur la musique, si on l'examine à l'aune des caractéristiques de la forme scolaire, ne renvoie pas à une rupture vis-à-vis de cette dernière. En effet, la porosité accrue de la forme scolaire ne signe pas pour autant une diminution de son emprise. Comme le suggère la volonté énoncée par les textes officiels de concilier le sensible et le code, il serait trop rapide de considérer cette évolution comme un recul de l'ambition formaliste - au sens de Durkheim - de l'éducation musicale au collège. S'il s'agit, comme on l'a vu précédemment, de privilégier l'«entrée sensible» dans les œuvres, il n'est pas pour autant question de se limi- ter à cette dimension de la perception et de la production artistique. Les élèves, avec l'aide de l'enseignant, doivent construire un savoir à partir de leurs «impressions immédiates » et, à terme, intérioriser des dispositions «cultivées» à l'égard de la musique. On retrouve ici le constat fait par I. Harlé sur l'introduction de l'enseignement de la technologie au collège : si celui-ci repose sur une volonté de s'appuyer sur le concret et la pratique dans le cadre de la massification de l'enseignement secondaire, il ne s'impose qu'à travers des formes scolaires instituées (Harlé, 2003).

Ce faisant, l'appui sur la sensibilité des élèves, destiné à les rendre acteurs des processus d'apprentissage, révèle des objectifs cognitifs beaucoup plus ambitieux que ceux associés au modèle de la «mémorisation-restitution » dans la mesure où il implique une appropriation des savoirs et une intériorisation des dispositions en jeu dans les apprentissages, et non une simple attitude de conformité. Moins les savoirs au centre des activités pédagogiques sont clairement définis (par exemple via une liste de notions de solfège que les élèves doivent maîtriser), dans le cadre d'un recours plus important à l'expérience ordinaire des élèves, plus les apprentissages supposent un «savoir-écouter » permettant de transmuer les contenus abordés en objet de connaissance.

\section{L'objectivation de la subjectivité musicale}

C'est véritablement à partir du programme de 1985 qu'est explicité le cheminement attendu des élèves pour convertir leur expérience subjective en une approche plus systématisée de la musique, mais la génération de programmes de la fin des années 1990, et plus encore celle de 2008, développent davantage les indications relatives à ce processus (les procédés pédagogiques prescrits étant au fil du temps de plus en plus détaillés : voir encadré). Si l'on reprend l'exemple des textes de 1995-1998, après une première phase d'écoute «globale et sensible», «sans souci prématuré d'analyse ou d'identification », qui se prolonge par un premier "commentaire subjectif », les élèves sont invités - et aidés pour cela par l'enseignant, ici «facilitateur», animateur et intermédiaire - à objectiver leurs impressions :

C'est seulement ensuite [après une première approche globale et sensible] que, guidés par le professeur dans des auditions fractionnées, les élèves pourront mieux cerner l'organisation des principales composantes musicales et synthétiser éventuellement leurs observations sous la forme de plans ou de schémas (Ministère de l'Éducation nationale, 1995). 
Si dans un premier temps de la séquence de cours, l'écoute, s'appuyant sur la subjectivité et les émotions, reste globale, le morceau étant appréhendé dans son unité, cette approche doit ensuite progressivement être transmuée en modalités d'appropriation d'ordre formel, à travers des procédés de décomposition, d'identification, de classement et de qualification. C'est donc un véritable processus de conversion des modes de perception et d'appréciation de la musique qui est attendu des élèves. L'extrait du texte d'accompagnement des programmes de $5^{\mathrm{e}}$ et $4^{\mathrm{e}}$ de 1997 suivant précise les étapes nécessaires à cette conversion :

Les écoutes suivantes permettront de s'approprier les éléments musicaux et les principes de composition essentiels retenus par le professeur. Les élèves seront ainsi conduits collectivement à mémoriser et reproduire les cellules, motifs, phrases, thèmes, etc., à les redécouvrir dans l'œuvre travaillée, puis dans l'audition d'autres extraits relevant d'esthétiques éventuellement différentes [...]. Pour chaque œuvre, la trace écrite figurant dans le cahier de l'élève s'élabore progressivement et collectivement. Elle peut prendre plusieurs formes éventuellement combinées : notes écrites, graphisme, fragment de partition, etc. (Ministère de l'Éducation nationale, 1997b).

Ce qui est décrit dans cet extrait renvoie à une opération de "décontextualisation-recontextualisation": les éléments faisant l'objet de la séquence de cours - à savoir des notions qui renvoient à une approche formelle de la musique consistant à en identifier la structure mélodique, harmonique ou rythmique («principes de composition", "cellules", etc.) - sont extraits de l'œuvre écoutée, sont travaillés pour eux-mêmes, puis replacés ensuite dans le contexte du morceau puis d'autres répertoires, processus relevant d'un rapport «scriptural-scolaire " au monde et au langage tel qu'il a été mis en évidence par B. Lahire $(1993,2008)$. Tout comme il le décrit au sujet du langage dans le cadre de l'apprentissage de la lecture et de l'écrit, l'éducation musicale, en décontextualisant la musique par rapport à ses usages ordinaires, la recontextualise en fait dans un autre système de relations, à savoir les différentes dimensions du langage musical formel ${ }^{14}$. La comparaison (oppositions, ressemblances, continuités...) entre répertoires hétérogènes, au principe d'un cheminement inductif vers le formalisme ${ }^{15}$, est centrale dans cette démarche. Ainsi, à travers la mise en lien de contenus musicaux à première vue dissemblables (du point de vue de l'époque ou du genre desquels ils relèvent, des instruments en présence, des thématiques auxquelles ils renvoient, etc.), il est attendu de l'élève qu'il puisse identifier des logiques formelles communes (par exemple la récurrence d'un motif mélodique, ou encore la présence d'une syncope rythmique). Dans cette logique, les programmes présentent ces procédés de mise en lien comme un moyen d'identifier et d'assimiler des éléments du langage musical en passant par l'« intuition musicale » des élèves, qui doivent sur cette base élaborer un savoir qui dépasse ce registre subjectif d'écoute.

Le cas de l'éducation musicale met tout particulièrement au jour le lien très étroit entre ce rapport au savoir attendu des élèves et la «disposition esthétique» telle qu'elle est décrite par P. Bourdieu (1979), disposition constitutive des mécanismes de distinction culturelle. D'une part, la «disposition esthétique » peut être ramenée à un processus de décontextualisation-recontextualisation, en tant qu'elle implique, selon P. Bourdieu, une rupture avec l'attitude ordinaire à l'égard du monde, avec ce qui relève de l'humain et de «la vie", afin d'adopter une posture ne se référant qu'à l'univers des œuvres d'art et de son histoire propre. D'autre part, cette disposition à l'égard de la culture repose, à l'instar des approches comparatives évoquées précédemment, sur des procédés de mise en relation, comme le souligne $P$. Bourdieu, qui décrit le «regard pur» sur les œuvres comme «un déplacement de l'intérêt du "contenu", personnages, péripéties, etc., vers la forme, vers les effets proprement artistiques qui ne s'apprécient que relationnellement, par une comparaison avec d'autres œuvres tout à fait exclusive de l'immersion dans la singularité de l'œuvre immédiatement donnée» (Bourdieu, 1979, p. 36). Dans cette mesure, les programmes de musique montrent bien en quoi le « rapport scriptural-scolaire" au savoir, visé par les textes officiels, constitue une forme rationalisée de la disposition esthétique, cette dernière étant généralement acquise par imprégnation et non par inculcation explicite.

L'opération de "décontextualisation-recontextualisation " attendue des élèves s'apparente à un processus d'objectivation du subjectif, dont le principe est particulièrement explicité dans les textes de 2008, notamment à travers un schéma (figure 1) retraçant à la fois le cheminement et les objectifs poursuivis à travers l'enseignement de la musique au collège :

Tout au long de sa scolarité au collège, l'élève mobilise et développe des capacités et attitudes spécifiques [...]. II apprend à tirer parti de la subjectivité de sa perception plutôt que d'en rester dépendant. [...] II apprend que sentiment et émotion sont les révélateurs d'une réalité complexe et permettent de comprendre les significations portées par la musique [...]. II apprend que l'émotion est un sentiment relatif, propre à chaque individu, qu'elle ne présume pas de l'objectivité des faits et doit 


\section{Figure 1. Schéma relatif à l'écoute musicale dans le programme de 2008}

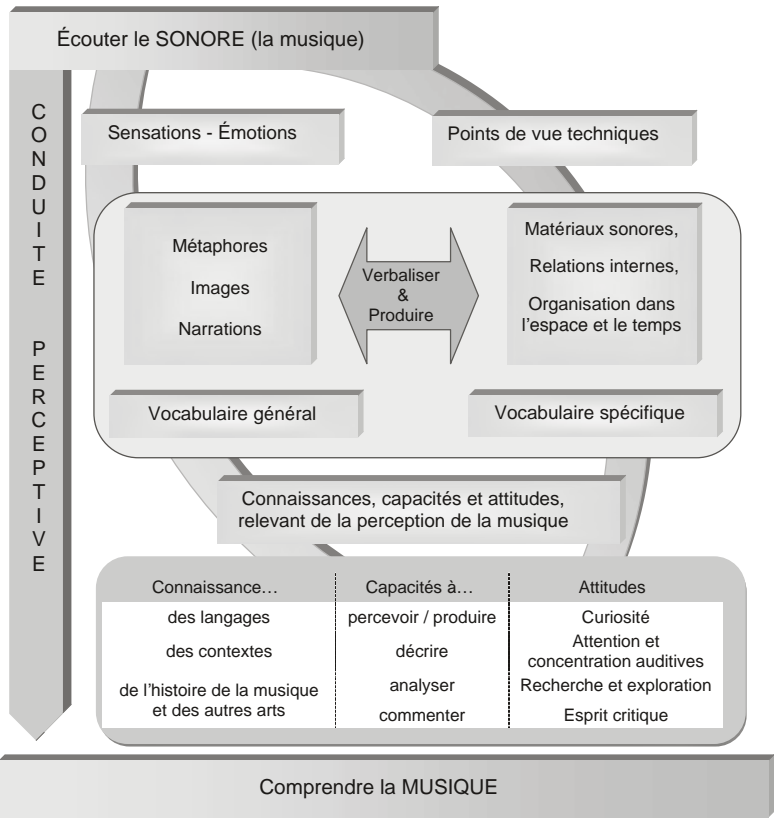

Source: Ministère de l'Éducation nationale, 2008.

donc être acceptée pour ce qu'elle est chez lui et chez les autres (Ministère de l'Éducation nationale, 2008).

Ces extraits du texte de 2008 décrivent bien, chacun à leur manière, ce processus d'objectivation du subjectif. Dans le premier, la «subjectivité», le «sentiment» et l'«émotion» sont présentés comme devant être mobilisés par l'élève, mais les "capacités " et «attitudes" attendues consistent justement à ce qu'il parvienne à les exploiter tout en s'en dégageant, en mettant ces dimensions à distance. Le deuxième extrait (figure 1) a pour ambition de décrire la "conduite perceptive", à savoir la manière dont les élèves doivent apprendre à percevoir la musique. Si les «sensations" et les "émotions» font partie intégrante du processus visé, elles ne constituent qu'un moyen au service d'une fin : la conversion du regard des élèves sur la musique pour qu'ils parviennent à une «compréhension » de la musique. Celle-ci, passant par l'élaboration de connaissances concernant le «langage», le "contexte», l' "histoire de la musique et des autres arts ", ou encore des capacités à "analyser » et à "commenter», fait directement écho à la «disposition esthétique» décrite par P. Bourdieu. On peut par ailleurs noter que, conformément à la logique défendue par les textes officiels depuis la fin des années 1970 , les «points de vue techniques» sont également présentés dans ce schéma comme mis au service du regard exigé des élèves sur la musique, et non comme une fin en soi.

Dans le contexte de la prise en compte de la sensibilité des élèves, les contenus de savoirs sont beaucoup plus subtils et difficiles à circonscrire. II ne s'agit plus de répertoires particuliers, ou de notions techniques, mais, in fine, d'un cheminement cognitif aboutissant à un "savoir-écouter». Deux processus concomitants et étroitement liés sont observables dans le corpus des textes officiels : d'une part, une moindre concentration sur les contenus, et notamment le répertoire des "canons musicaux » qui renvoie au décloisonnement entre culture scolaire et "culture libre", et, d'autre part, une focalisation sur la transmission de compétences d'écoute. Ce double processus, qui aboutit à un nouveau type de formalisme dans les curricula que décrit le schéma présenté plus haut (figure 1), et dans lequel le statut accordé aux grandes œuvres décline, peut être analysé une fois de plus en s'appuyant sur la description que fait $B$. Bernstein du code intégré : "Les différents contenus du savoir sont subordonnés à une certaine idée qui les rapproche les uns des autres et, par suite, réduit l'autorité propre de chacun de ces savoirs envisagés séparément » (Bernstein, 1975, p. 283). Ainsi, cette citation permet de toucher du doigt le lien entre importance des compétences relatives à des procédés de mises en relation et moindre poids des contenus.

Le rapport des programmes aux canons musicaux évolue en effet considérablement. Ceux-ci sont, jusqu'aux années 1960, au cœur des visées de l'éducation musicale, qui doit amener les élèves à les connaître et les reconnaître, avec par exemple l'idée qu'il faut se baser, en chant, sur les "mélodies simples des maîtres" (Ministère de l'Éducation nationale, 1938a), qu'«il faut mettre les élèves exclusivement en présence de belle musique et proscrire la médiocrité " (Ministère de l'Éducation nationale, 1938b), ou encore qu'«il n'est pas permis d'ignorer » certaines œuvres comme la musique religieuse de Palestrina, de Luther et de Bach. Les schèmes de perception et d'appréciation relevant de la disposition esthétique, "seule manière socialement tenue pour "convenable" d'aborder les objets socialement désignés comme œuvres d'art » (Bourdieu, 1979, p. 29), sont en jeu dans cette transmission des canons. Néanmoins, ils n'en constituent pas l'objectif premier (centré sur la connaissance des œuvres et des compositeurs), et les savoirs relatifs au solfège renvoyant à une appréhension formelle de la musique sont étudiés indépendamment. 
À partir des années 1970, les répertoires évoqués ne se limitent plus aux canons de la musique "savante", mais surtout l'injonction centrale en la matière consiste désormais à étudier la plus grande diversité possible de registres musicaux : c'est ce qu'énonce par exemple le programme de 1985 : «Le champ d'investigation doit être aussi large que possible [...]. Le répertoire musical doit être utilisé dans toute son étendue et sa diversité" (Ministère de l'Éducation nationale, 1985). Dans ce cadre, les modalités de perception et d'appréciation de la musique inhérentes à la "disposition esthétique» deviennent centrales et sont destinées à être appliquées à tous les répertoires musicaux, qu'ils relèvent ou non de la musique "savante» la plus consacrée. C'est d'ailleurs via cette diversité musicale et les comparaisons qu'elle offre que ces compétences d'écoute doivent être acquises.

Ce constat fait fortement écho au recentrage des programmes scolaires autour de la transmission de compétences (Ropé \& Tanguy, 1994; Clément, 2013) au détriment de l'étude des grandes œuvres, et notamment en français, discipline dans laquelle ce phénomène est relié par la littérature sociologique aussi bien à des mutations culturelles comme le développement des communications «de masse » qu'à la diversification importante du public de l'enseignement secondaire. Ainsi, ce "savoirécouter » est comparable au "savoir-communiquer » dont L. Demailly (1985) a montré l'importance dans l'enseignement du français à partir des années $1980^{16}$, analyse qui rejoint celle faite par $V$. Isambert-Jamati et M.-F. Grospiron concernant les instructions officielles de 1981 relatives à l'enseignement du français au lycée, dans lesquelles "on parle au premier chef de "former des capacités qui permettent de communiquer et de s'exprimer dans la langue d'aujourd'hui" " tandis que l'étude des grands auteurs perd de son importance (Isambert-Jamati \& Grospiron, 1984).

\section{L'objectif de la "domestication" des pratiques d'écoute des élèves}

On peut par conséquent estimer que l'ouverture de l'école à l'expérience ordinaire des élèves relève d'une «hyperscolarisation implicite» (Bonnéry, 2007, p. 71). En effet, dans l'optique des textes officiels, les élèves doivent être eux-mêmes en mesure, de manière autonome et spontanée, de scolariser leurs expériences ordinaires pour qu'elles deviennent objets de savoir, ce qui revient paradoxalement à donner davantage de poids à l'objectif de l'acquisition d'un regard formel sur la musique. On peut faire ici le parallèle avec l'évolution de la forme scolaire qui s'est opérée à travers le développement des écoles mutuelles. Avec ces dernières, la discipline doit être intériorisée par les élèves, comprise et acceptée et pas seulement appliquée (Vincent, Lahire \& Thin, 1994; Vincent, 1980) ${ }^{17}$. De même, on peut envisager les évolutions des programmes de musique comme une variation comparable de la forme scolaire : les cheminements intellectuels attendus des élèves doivent être non seulement suivis par les élèves, mais également intériorisés et maîtrisés, jusqu'à devenir une «seconde nature».

Loin de se cantonner à la sphère scolaire, cette ambition concerne les pratiques d'écoute des élèves en général, y compris hors l'école. L'objectif est donc de «redresser » durablement les dispositions à l'égard de la musique des élèves, développées en grande partie hors du cadre scolaire. À terme, l'élève doit incorporer les rapports à la musique prescrits par l'école, ceux-ci devant devenir «spontanés", comme l'énonce le texte de 1985 : «Une oreille bien éduquée est une oreille qui a appris à mettre spontanément de l'ordre dans la complexité brute des matériaux sonores. Elle est prête à assimiler des éléments de couleur, de forme et de style qui sont au fondement de la culture musicale" (Ministère de l'Éducation nationale, 1985).

Les élèves doivent, en d'autres termes, se défaire des schèmes d'appréciation développés hors de l'école pour parvenir à un regard formel sur les œuvres, seul considéré comme valable ou adéquat par les programmes. Cette ambition de redresser les pratiques des élèves, loin de se limiter à la sphère strictement scolaire, vise les situations d'écoute extra-scolaires :

La musique tient une place essentielle dans la vie des élèves aujourd'hui. II revient à l'éducation musicale de donner les moyens de s'approprier ce mode d'expression et les aider à acquérir des capacités de discernement et un esprit d'ouverture (Ministère de l'Éducation nationale, 1995).

II [l'élève] apprend à transposer ses connaissances et compétences vers des musiques qu'il écoute de sa propre initiative. [...] Si l'élève dispose aujourd'hui de tous les moyens possibles pour écouter, l'éducation musicale lui apprend à en tirer parti. Sur des supports variés, elle entraîne sa perception à construire des connaissances et à susciter des émotions nouvelles. Elle l'amène à identifier les caractéristiques constitutives de ce qu'il entend afin de construire un avis personnel argumenté. II développe ses capacités de jugement et son esprit critique. II effectue progressivement des choix personnels dans le vaste éventail des musiques qu'il peut écouter (Ministère de l'Éducation nationale, 2008).

II s'agit bien de «domestiquer» les pratiques d'écoute 
juvénile, dans la mesure où l'empreinte de l'enseignement scolaire de la musique est destinée à être intériorisée, à façonner les habitudes des élèves en dehors du monde scolaire, tout comme l'usage de l'écrit modifie en profondeur les «modes de pensée» des sociétés alphabétisées. Cette influence ne se limite pas aux situations dans lesquelles intervient l'écrit, mais produit des habitudes durables en termes d'itinéraire de pensée, ce qui amène J. Goody à parler de «domestication» de la «pensée sauvage» (Goody, 1979). De la même manière, les procédés utilisés dans le cadre de l'enseignement scolaire de la musique sont destinés à forger chez eux des schèmes pérennes de perception et d'appréciation de la musique qui relèvent de dispositions «cultivées » à l'égard de la musique, et qui leur permettent d'établir eux-mêmes des hiérarchies musicales n'étant plus données d'emblée par les instructions officielles.

La référence centrale à la sensibilité des élèves perceptible dans les textes officiels depuis les années 1980 se conjugue donc à l'intériorisation de la «disposition esthétique », signalant un approfondissement de l'objectif de la transmission d'un rapport formel au savoir. $\mathrm{Ce}$ faisant, l'éducation musicale suit une tendance générale affectant la forme scolaire, qui s'est recentrée sur des dispositifs exigeant la compréhension et l'appropriation des savoirs par les élèves.

L'exemple de l'enseignement scolaire de la musique met en exergue en quoi cette évolution a partie liée avec les transformations des mécanismes de distinction soulignées par la littérature sociologique (Coulangeon, 2010, 2011 ; Donnat, 1994 ; Peterson \& Simkus, 1992), dans le contexte d'un brouillage des frontières symboliques entre «savant » et «populaire » remettant en question la définition traditionnelle de la légitimité culturelle, centrée autour de la familiarité avec les répertoires culturels «savants». Ainsi, ces analyses s'accordent sur le constat selon lequel les attitudes culturelles des classes supérieures se caractériseraient aujourd'hui davantage par l'hétérogénéité de leurs registres de consommation culturelle que par leur appétence pour les répertoires «savants», signalant ce faisant une valorisation sociale de l'éclectisme culturel qui fait pendant à l'injonction à la diversité musicale présente dans les programmes depuis les années 1980. Dans ce cadre, O. Donnat et P. Coulangeon signalent l'importance accrue des modalités d'appropriation des biens culturels dans les mécanismes de distinction culturelle, qui fait également écho au recentrage des objectifs de l'éducation musicale autour de la transmission de schèmes de perception et d'appréciation de la musique relevant de la «disposition esthétique» : c'est autant, si ce n'est plus, à travers la manière d'écouter de la musique qu'il s'agit de se distinguer, que par les répertoires musicaux appréciés ou écoutés.

\section{CONCLUSION}

Les évolutions du curriculum de l'enseignement de la musique au collège que nous avons soulignées dans cet article signalent moins une remise en cause de la forme scolaire qu'une variation de cette dernière, puisque le rapport «scriptural-scolaire» au monde qu'elle suppose est mis au centre des apprentissages. À la suite de G. Vincent, B. Lahire et D. Thin (1994, p.45-46), on peut donc interpréter l'ouverture du curriculum à l'expérience ordinaire des élèves comme une extension de l'emprise de la forme scolaire, suffisamment hégémonique - à l'intérieur, mais aussi en dehors de l'institution scolaire - pour que la scolarisation de formes culturelles extérieures à l'école soit possible. Dans ce cadre, on peut reprendre l'analyse que proposait É. Durkheim du système scolaire dans L'évolution pédagogique en France, selon lequel on voit «passer [l'école] de formalisme en formalisme » (Durkheim, [1938] 1990, p.61). Récusant une approche jargonneuse et trop déconnectée de l'«activité musicale réelle en s'appuyant sur la sensibilité des élèves, l'éducation musicale n'en abandonne pas moins - et approfondit même au cours de la période étudiée - une visée formaliste constitutive de la «mise en forme scolaire» de la musique.

En outre, l'éducation musicale, en se centrant davantage sur la transmission d'un rapport à la culture qui relève de la «disposition esthétique» - relativement autonome par rapport aux contenus transmis - prend acte et contribue à la diffusion des transformations de la culture légitime. Ce faisant, l'éducation musicale peut être appréhendée comme une discipline permettant tout particulièrement de toucher du doigt le renouvellement du lien étroit entre culture scolaire et culture légitime, qui pose sous d'autres formes la question des inégalités sociales d'appropriation des savoirs scolaires.

Florence Eloy florence_eloy@yahoo.fr Université Paris 8-Vincennes-Saint-Denis, CIRCEFT-ESCOL, CEMS/IMM-EHESS 


\section{NOTES}

1 Le champ recouvert par cette expression est très hétérogène et renvoie, dans le cadre de cet article, davantage à la vulgate des «méthodes actives » qui lui est liée qu'à la grande variété d'auteurs et d'élaborations théoriques de ce courant.

2 C'est sur cet aspect cognitif de la forme scolaire, mis en exergue par B. Lahire (1993), que nous nous centrerons dans cet article.

3 Si cette discipline relève des matières obligatoires au collège, et renvoie donc à des savoirs estimés indispensables à ce niveau du système éducatif, la faiblesse des coefficients et des volumes horaires qui lui sont affectés sont autant de signes de la reconnaissance limitée dont elle bénéficie et de son poids limité dans les processus de sanction et d'orientation scolaire.

4 Selon les données de l'enquête L'enfance des loisirs, à la fin du collège, en 2006, plus de la moitié des élèves écoute de la musique quotidiennement $(58 \%)$, pourcentage qui progresse au fur et à mesure de l'avancée dans l'adolescence (Octobre, Détrez, Mercklé et al., 2010).

5 Pour plus détail sur le corpus de textes officiels sur lequel s'appuie cet article, voir l'encadré. L'analyse présentée dans cet article est issue d'un travail de thèse sur les tensions et les porosités entre modalités de scolarisation de la musique et pratiques culturelles juvéniles (Eloy, 2012), dans le cadre duquel ce corpus est confronté à d'autres types de données permettant de ne pas limiter la réflexion à la dimension du «curriculum formel » (Isambert-Jamati, 1990). D'une part, il s'agit d'une enquête de terrain dans des établissements secondaires à recrutement socio-scolaire contrasté. Ce travail de terrain a donné lieu à des observations de pratiques d'enseignement de la musique dans une dizaine de classes de $3 e$, complétées par des entretiens avec des professeurs de musique et des élèves. D'autre part, nous avons également procédé à une analyse secondaire des données de l'enquête L'enfance des loisirs (Octobre, Détrez, Mercklé et al., 2010) du ministère de la Culture et de la Communication sur les univers culturels des enfants et des adolescents, comportant notamment des questions sur les goûts et pratiques en matière de musique, ainsi que sur les enseignements artistiques.

6 Pour plus de détail sur ce point, voir d'autres publications dans lesquelles nous avons plus précisément exploré la question de l'évolution des répertoires musicaux mobilisés dans le cadre de l'enseignement scolaire de la musique (Eloy, 2010, 2014).

7 Souligné par nous. Tous les passages de citation en italique le sont également dans l'ensemble de cet article.

8 C'est ce qui transparaît clairement dans ces extraits d'une note du 3 mai 1968 de Marcel Landowski, alors directeur de la musique au ministère des Affaires culturelles (fonction qu'il exerce de 1966 à 1975 avant d'être nommé inspecteur général de la musique au ministère de l'éducation nationale), concernant les programmes de $2 \mathrm{de}$, $1^{\text {re e }}$ et terminale section $A$ ainsi que des épreuves de musique du baccalauréat, rapportés par $\mathrm{O}$. Tripier-Mondancin : «M'inspirant de cette idée qui me paraît essentielle, "les disciplines de la sensibilité sont aussi importantes pour la formation d'un homme que les disciplines de la connaissance", j'ai proposé un programme faisant appel surtout à la participation des élèves à diverses actions musicales, c'est-à-dire à la créativité, à l'improvisation, à la connaissance pratique des éléments sonores mis à notre disposition par les sciences, à la réflexion enfin sur les divers aspects psychiques, sociaux et historiques du phénomène musical, j'ai donc été amené à ne pas accepter le projet présenté par l'inspecteur général Favre, projet basé sur le solfège, l'analyse harmonique et l'histoire de la musique [...]. La mutation profonde, la révolution presque qu'implique le programme de baccalauréat option musique tel qu'il a été décidé, demande que l'homme qui sera chargé de le faire appliquer soit un homme profondément convaincu de la nécessité de sortir des habitudes actuelles que nous considérons comme néfastes et qu'il soit l'apôtre de la pédagogie nouvelle [...]. La nouvelle orientation pédagogique ne doit pas être seulement appliquée aux classes terminales, option musique, mais bien à l'ensemble de l'enseigne- ment musical dans l'enseignement général depuis les cours préparatoires du cycle primaire» (Landowski, cité par Tripier, 2008, p. 225).

9 Ce genre d'assertion contraste avec la vision de la culture juvénile qui transparaissait dans les programmes de l'immédiat après-guerre, où les élèves sont plutôt décrits comme ayant peu de connaissances concrètes de la musique, la mission de l'éducation musicale étant de les sensibiliser à ce domaine artistique : "L'enseignement musical n'a pas seulement pour objet d'amener les élèves à chanter en chœur; il tend aussi à leur donner le goût de la musique. [...] L'essentiel sera que les élèves comprennent la richesse et la variété d'un moyen d'expression représenté pour eux trop souvent par des mots et des noms sans substances " (Ministère de l'Éducation nationale, 1944).

10 Les activités de création sont introduites dans le curriculum de la discipline avec les programmes de 1985-1987. Elles peuvent consister, par exemple, à réécrire le texte d'une chanson déjà existante, ou à y ajouter des parties de chant ou d'instruments. Sur ce type d'activités, les programmes incitent également les enseignants à choisir des chansons correspondant à la culture musicale des élèves : «La création de chanson permet aux élèves de s'exprimer avec leurs mots, avec leurs musiques tout en intégrant les acquis du cours d'éducation musicale" (Ministère de l'Éducation nationale, 1997a).

11 Dans le cadre de cet article, nous n'avons pu citer l'ensemble des extraits des textes officiels se référant aux procédés pédagogiques et aux visées de l'enseignement de la musique au collège que nous mettons en exergue; sauf mention inverse, les tendances identifiées se retrouvent dans tous les programmes à partir des années 1970.

12 La diffusion de ces conceptions pédagogiques durant les dernières décennies a été largement mise en évidence à travers des travaux portant sur des segments variés du système éducatif, notamment dans le cadre de l'équipe ESCOL (Charlot, Bautier \& Rochex, 1992; Bautier \& Rochex, 1997; Bonnéry, 2007; Lahire, 1993; Rochex \& Crinon, 2011).

13 Si l'élargissement des références musicales énoncées dans les programmes est sélectif et concerne les pans les plus consacrés des répertoires dits "populaires", il n'en est pas moins notable. Par exemple, le schéma illustrant le «domaine des styles» à balayer avec les élèves présenté par les programmes de 2008 cite comme faisant partie du "vocabulaire de référence" les "comédies musicales », les «musiques de film », le «jazz », les «musiques actuelles» ou encore le «rock» sur la période du $x x^{e}$ siècle, au côté des «musiques savantes et usant de nouveaux matériaux, langages et technologies » (Ministère de l'Éducation nationale, 2008).

14 «En se plaçant dans une telle position par rapport au langage [celle de la logique scripturale-scolaire], l'élève découvre un monde de relations tout à fait inédit. Les divers exercices scolaires le forcent à saisir les différents éléments du langage comme les parties d'un système complexe de relations qu'il ignorait jusqu'à là. La décontextualisation des éléments du langage opérée à l'école est bien une recontextualisation dans de nouveaux contextes (paradigmes et systèmes de relations entre les éléments des différents paradigmes) qui ne sont pas plus artificiels que les contextes ordinaires " (Lahire, 2008, p. 55).

15 Cela s'inscrit plus globalement dans une tendance au développement, mis en exergue par S. Bonnéry (2009, p. 21-22), des dispositifs pédagogiques relevant de logiques comparatives et inductives.

16 L. Demailly estime ainsi que le développement des communications de masse a conduit à une mutation de l'enseignement du français, dont les objectifs sont passés d'un raffinement élitaire à un «savoircommuniquer »: «En matière d'éducation langagière, le développement des communications de masse valorise, aux dépens de la recherche d'un raffinement élitaire, un "savoir-communiquer", transclassiste et discret. L'école est censée maintenant fournir aux enfants une compétence linguistique polyvalente d'intervention et de communication» (Demailly, 1985, p.110, souligné par l'auteur).

17 "La transformation de l'écolier discipliné et "dressé" en écolier "raisonnable", qui suppose un autre rapport aux règles impersonnelles, 
va s'opérer tout d'abord à l'intérieur des écoles mutuelles. [...] Tout ce qui est enseigné doit être expliqué, la discipline ne doit pas être subie mais comprise et acceptée. Si l'élève comprend les règles, il se les approprie par lui-même et pratique une sorte d'auto-discipline, un self-government » (Vincent, Lahire \& Thin, 1994, p. 34-35).

\section{BIBLIOGRAPHIE}

BANTIGNY L. (2008). «Les deux écoles. Culture scolaire, culture de jeunes: genèse et troubles d'une rencontre, 19601980 ». Revue française de pédagogie, no163, p. 15-25.

BARRÈRE A. \& JACQUET-FRANCILLON F. (2008). «La culture des élèves : enjeux et questions ». Revue française de pédagogie, no163, p.5-13.

BAUTIER É. \& RAYOU P. (2009). Les inégalités d'apprentissage. Programmes, pratiques et malentendus scolaires. Paris : PUF.

BAUTIER É. \& ROCHEX J.-Y. (1997). «Apprendre : des malentendus qui font la différence». In J.-P. Terrail, La scolarisation de la France. Critique de l'état des lieux. Paris : La Dispute, p. 105-122.

BERNSTEIN B. (1975). Langage et classes sociales. Codes socio-linguistiques et contrôle social. Paris : Éd. de Minuit.

BONNÉRY S. (2007). Comprendre l'échec scolaire. élèves en difficultés et dispositifs pédagogiques. Paris : La Dispute.

BONNÉRY S. (2009). «Scénarisation des dispositifs pédagogiques et inégalités d'apprentissage ». Revue française de pédagogie, no167, p.13-23.

BOURDIEU P. (1967). "Systèmes d'enseignement et systèmes de pensée". Revue internationale des sciences sociales, vol.119, n॰3, p.367-388.

BOURDIEU P. (1979). La Distinction. Critique sociale du jugement. Paris : Éd. de Minuit.

BOURDIEU P. (1980). Le sens pratique. Paris : Éd. de Minuit.

BOURDIEU P. (2004). Esquisse pour une auto-analyse. Paris : Raisons d'agir.

BOURDIEU P. \& PASSERON J.-C. (1964). Les héritiers. Les étudiants et la culture. Paris : Éd. de Minuit.

CHARLOT B., BAUTIER É. \& ROCHEX J.-Y. (1992). École et savoir dans les banlieues... et ailleurs. Paris : Armand Colin.

CLÉMENT P. (2013). Réformer les programmes pour changer l'école? Une sociologie historique du champ du pouvoir scolaire. Thèse de doctorat, sociologie, université de Picardie-Jules-Verne.

COULANGEON P. (2010). «Les métamorphoses de la légitimité». Actes de la recherche en sciences sociales, no181-182, p. 88-105.

COULANGEON P. (2011). Les métamorphoses de la distinction. Inégalités culturelles dans la France d'aujourd'hui. Paris : Grasset.

DEMAILLY L. (1985). “Contribution à une sociologie des pratiques pédagogiques». Revue française de sociologie, vol. 26, no1, p.96-119.

DONNAT O. (1994). Les Français face à la culture : de l'exclusion à l'éclectisme. Paris : La Découverte.

DURKHEIM É. ([1938] 1990). L'évolution pédagogique en France. Paris : PUF.
ELOY F. (2010). «Esthétisation du populaire, popularisation du savant : les stratégies d'utilisation de la culture juvénile dans le cadre des cours d'éducation musicale au collège". In S. Octobre \& R. Sirota, Actes du colloque "Enfance et cultures : regards des sciences humaines et sociales». En ligne : <http://www.enfanceetcultures.culture.gouv.fr/?id page $=$ colloque\&lang=fr $>$ (consulté le 15 octobre 2013).

ELOY F. (2012). Apprendre à écouter la musique. Culture légitime, culture scolaire et cultures juvéniles. Thèse de doctorat, sociologie, EHESS.

ELOY F. (2014, à paraître). "L'émergence de la norme de I'“éclectisme éclairé" dans l'enseignement de la musique au collège». In S. Bonnéry, Supports pédagogiques et inégalités scolaires. Études sociologiques. Paris : La Dispute.

GOODY J. (1979). La raison graphique. La domestication de la pensée sauvage. Paris : Éd. de Minuit.

HARLÉ I. (2003). «L'introduction d'une culture technique au collège : une analyse socio-historique ". Revue française de pédagogie, n¹44, p.95-103.

ISAMBERT-JAMATI V. (1970). Crises de la société, crises de l'enseignement. Sociologie de l'enseignement secondaire français. Paris : PUF.

ISAMBERT-JAMATI V. (1990). Les savoirs scolaires. Enjeux des contenus d'enseignement et de leurs réformes, Paris : éditions universitaires.

ISAMBERT-JAMATI V. \& GROSPIRON M.-F. (1984). «Types de pédagogie du français et différenciation sociale des résultats. L'exemple du "travail autonome" au deuxième cycle long ". Études de linguistique appliquée, n॰54, p.69-97.

JOHSUA S. (1999). L'école, entre crise et refondation. Paris : La Dispute.

LAHIRE B. (1993). Culture écrite et inégalités scolaires. Sociologie de l'«échec scolaire» à l'école primaire. Lyon : Presses universitaires de Lyon.

LAHIRE B. (2004). La culture des individus. Dissonances culturelles et distinction de soi. Paris : La Découverte.

LAHIRE B. (2005). "Misère de la division du travail sociologique : le cas des pratiques culturelles adolescentes ". Éducation et sociétés, vol. 16, n², p. 129-136.

LAHIRE B. (2008). La raison scolaire. école et pratique d'écriture, entre savoir et pouvoir. Rennes : Presses universitaires de Rennes.

OCTOBRE S., DÉTREZ C., MERCKLÉ P. \& BERTHOMIER N. (2010). L'enfance des loisirs. Trajectoires communes et parcours individuels de la fin de l'enfance à la grande adolescence. Paris : DEPS, Ministère de la Culture et de la Communication.

PASQUIER D. (2005). Cultures lycéennes. La tyrannie de la majorité. Paris : Autrement. 
PLAISANCE É. (1986). L'enfant, la maternelle, la société. Paris : PUF.

PETERSON R. A. \& SIMKUS A. (1992). «How musical tastes mark occupational status groups». In M. Fournier \& M. Lamont, Cultivating Differences. Symbolic Boundaries and the Making of Inequality. Chicago; Londres : The University of Chicago Press, p.152-186.

RAYOU P. (2000). «L'enfant au centre. Un lieu commun pédagogiquement correct». In J.-L. Derouet (dir.), L'école dans plusieurs mondes. Bruxelles : De Boeck, p. 245-290.

ROCHEX J.-Y. \& CRINON J. (dir.). (2011). La construction des inégalités scolaires : au cœur des pratiques et des dispositifs d'enseignement. Rennes: Presses universitaires de Rennes.

ROPÉ F. \& TANGUY L. (dir.) (1994). Savoirs et compétences : de l'usage de ces notions dans l'école et l'entreprise. Paris : L'Harmattan.
SOHN A.-M. (2001). Âge tendre et tête de bois : histoire des jeunes des années 1960. Paris: Hachette.

TRIPIER-MONDANCIN O. (2008). L'Éducation musicale au collège depuis 1985. Valeurs déclarées par des enseignants d'Éducation musicale, genèse. Thèse de doctorat, sciences de l'éducation, université de Toulouse 2-Le Mirail.

VINCENT G. (1980). L'École primaire française. Étude sociologique. Lyon : Presses universitaires de Lyon; Paris : éd. de la Maison des sciences de l'homme.

VINCENT G., LAHIRE B. \& THIN D. (1994). «Sur I'histoire et la théorie de la forme scolaire". In G. Vincent (dir.), L'éducation prisonnière de la forme scolaire? Scolarisation et socialisation dans les sociétés industrielles. Lyon : Presses universitaires de Lyon, p. 11-48.

\section{TEXTES RÉGLEMENTAIRES}

MINISTÈRE DE L'ÉDUCATION NATIONALE (1938a). Horaires et programmes du second degré. Arrêtés du 30 août 1937 et du 11 avril 1938 fixant les programmes du 2d degré. Paris : Vuibert.

MINISTÈRE DE L'ÉDUCATION NATIONALE (1938b). Instructions du 30 septembre 1938 relatives à l'application des arrêtés du 30 août 1937 et du 11 avril 1938 fixant les programmes de l'enseignement du $2 d$ degré. Paris : Vuibert.

MINISTÈRE DE L'ÉDUCATION NATIONALE (1944). «Arrêté du 21 septembre 1944 (annexe). Enseignement. Horaires et programmes (Histoire.-Géographie.-Civilisation et littératures étrangères.-Éducation musicale et chant choral) ». Bulletin officiel de l'Éducation nationale, n॰ 4 du 12 octobre 1944.

MINISTÈRE DE L'ÉDUCATION NATIONALE (1977a). «Arrêté du 17 mars 1977. Programme de l'éducation musicale $6^{e}$ et $5^{\mathrm{e}}$ ». Bulletin officiel de l'Éducation nationale, no11 du 24 mars 977.

MINISTÈRE DE L'ÉDUCATION NATIONALE (1977b). «Circulaire no77-165 du 29 avril 1977. Enseignement de l'Éducation artistique dans les collèges ». Bulletin officiel de l'Éducation nationale, $\mathrm{n}^{\circ} 22$ ter du 9 juin 1977.

MINISTÈRE DE L'ÉDUCATION NATIONALE (1985). «Arrêté du 14 novembre 1985 fixant les programmes des classes de collège ». Bulletin officiel de l'Éducation nationale, n॰44 du 12 décembre 1985.

MINISTÈRE DE L'ÉDUCATION NATIONALE (1995). «Arrêté du 22 novembre 1995. Programme du cycle d'adaptation.

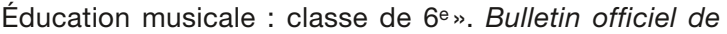
l'Éducation nationale, no 48 du 28 décembre 1995.

MINISTÈRE DE L'ÉDUCATION NATIONALE (1997a). «Arrêté du 10 janvier 1997. Programmes du cycle central du collège. Éducation musicale». Bulletin officiel de l'Éducation nationale, hors série n॰1 du 13 février 1997.

MINISTÈRE DE L'ÉDUCATION NATIONALE (1997b). «Complément de l'arrêté du 10 janvier 1997. Accompagnement des programmes du cycle central de $5^{\mathrm{e}}$ et $4^{\mathrm{e}}$ ». Bulletin officiel de l'Éducation nationale, hors série no1 du 13 février 1997.

MINISTÈRE DE L'ÉDUCATION NATIONALE (1998). «Accompagnement des programmes de $3 \mathrm{e}$ ». Enseignements artistiques, arts plastiques, éducation musicale : programmes et accompagnement. Paris : CNDP.

MINISTÈRE DE L'ÉDUCATION NATIONALE (2008). «Programmes du collège. Programmes de l'enseignement d'éducation musicale». Bulletin officiel de l'Éducation nationale, numéro spécial no 6 du 28 août 2008. 